\title{
DUROZIEZ'S SIGN IN NORMAL SUBJECTS AND IN PATIENTS WITH ARTERIAL HYPERTENSION
}

\author{
With Special Reference to Its Relation to Capillary Pulsation and \\ the Forward Flow of Blood During Diastole
}

\author{
By SAMUEL BROWN AND BENJAMIN ALEXANDER 1 \\ (From the Medical Service and Medical Research Laboratories of the Beth Israel Hospital and \\ the Department of Medicine, Hariard University Medical School, Boston)
}

(Received for publication November 22, 1934)

Blumgart and Ernstene (1) recently have shown that a diastolic murmur heard over the larger peripheral arteries, the so-called Duroziez's sign, may be produced by two opposite mechanisms. By altering the direction of pressure of the stethoscope bell, by subjecting the arm to varying temperatures of water, and by applying a subdiastolic pressure below the site of auscultation they were able to differentiate between the diastolic murmur heard in aortic insufficiency and that heard in individuals with peripheral vasodilatation. It was shown that the diastolic murmur in aortic insufficiency is produced by a backward flow of blood toward the heart due to incompetent aortic valve action; in peripheral vasodilatation the murmur was found to be due to an increase in the forward flow of blood toward the periphery. Such vasodilatation is commonly present in thyrotoxicosis and febrile states or may be induced in normal individuals by the local application of heat.

The state of dilatation of the peripheral vascular bed determines, therefore, the presence or absence of Duroziez's sign when this sign is produced by an increased forward flow of blood (1). Lewis (2) has shown that the state of the peripheral vascular bed determines the presence or absence of capillary pulsation. This author concluded that capillary pulsation is a physiological phenomenon occurring in skin or mucous membrane whenever the arterioles are sufficiently dilated, and suggested that the presence of capillary pulsation may be used as an index of the dilatability of the peripheral arterioles. Since the type of Duroziez's sign due to forward flow of blood likewise depends on the state of the peripheral

\footnotetext{
1 James Jackson Cabot Student Research Fellow at Harvard Medical School.
}

minute vessels it seemed of interest to study these two signs in various normal and diseased states.

The purpose of this investigation is (1) to study, in health and vascular disease, the incidence of Duroziez's ${ }^{2}$ sign artificially produced by immersing the arm in water at $114^{\circ} \mathrm{F}$; ; (2) to correlate this sign with other peripheral vascular signs such as capillary pulsation, blood pressure and pulse pressure; (3) to evaluate Duroziez's sign as an index of the dilatability of the peripheral vascular bed.

\section{METHOD}

Three groups of subjects were studied: (1) Subjects with no clinical evidence of cardiovascular disease; (2) subjects with definite arterial hypertension; (3) a small miscellaneous group consisting of other vascular and associated diseases. No patients with spontaneous Duroziez's sign or with clinical evidence of aortic valvular disease were included in the study.

Each experiment lasted about ten minutes and all observations were checked by two observers. The pulse rate, body temperature, room temperature, systolic and diastolic blood pressures, and presence or absence of capillary pulsation were determined and recorded. By means of a Peabody bell stethoscope connected to two sets of ear pieces (Figure 1) a spontaneous diastolic murmur was sought over the antecubital space. The subject's forearm was then immersed in a water bath at $114^{\circ} \mathrm{F}$. and with stethoscope bell applied as before, the time of appearance of a diastolic murmur was noted. In general, if a diastolic murmur appeared it did so within three minutes. When Duroziez's sign failed to appear,

\footnotetext{
2 Throughout the remainder of the paper "Duroziez's sign" indicates Duroziez's sign due to the forward flow of blood toward the periphery.
} 
auscultation during immersion was continued for five minutes. At the end of this time observations on the capillary pulsation were again made and systolic and diastolic blood pressures again measured. from a sharp loud to a low muffled sound. Care was taken to maintain the temperature of the water at $114^{\circ} \mathrm{F}$. by adding hot water when necessary.

The capillary pulsation immediately before and

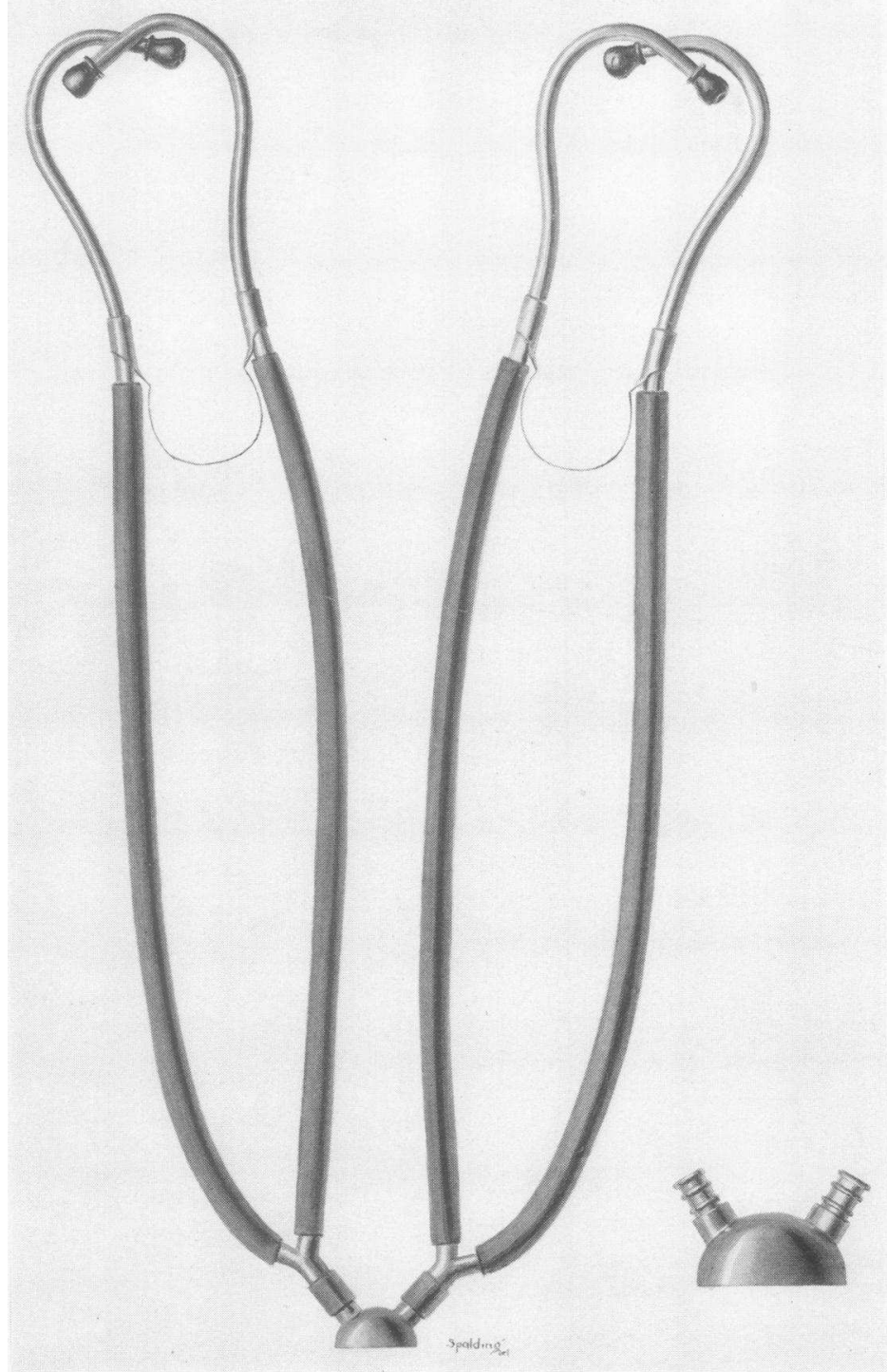

Fig. 1. Arrangement of Stethoscope

Peabody Bell.

The standard mercury sphygmomanometer with cuff $14 \mathrm{~cm}$. wide was used. All measurements were made within the minute immediately preceding and following the immersion of the forearm in hot water. The arterial diastolic pressure reading was taken at the point of change after immersion was sought by pressing with a glass slide over the pads of the distal phalanges of the fingers. Each observation was checked by two observers, one of whom counted the alternate waxing and waning of the pulsation, while the other checked this with the patient's pulse. In 
each ,case the observer's own pulse was eliminated as a possible determining factor.

Only diastolic murmurs that could be maintained indefinitely and that filled or practically filled the entire duration of diastole were considered typical of Duroziez's sign. In the older age groups a definite diastolic murmur produced in the usual manner and heard during many successive cycles generally lasted only through approximately three-fourths of diastole. Such murmurs were included as positive. Throughout the study the patient was at rest. Enough observations were made on different days to be certain of the constancy of findings for each patient.

\section{OBSERVATIONS}

A. Duroziez's sign in normal subjects ${ }^{3}$ and in patients with arterial hypertension

$V$ ariation with age. In subjects with no evidence of cardiovascular disease the incidence of the artificially produced Duroziez's sign is greatest in young subjects and decreases with advancing years (Figure 2). In subjects between the ages of 20 and 40 years, Duroziez's sign was practically always obtainable; in middle age, 40 to 60 years, the incidence dropped sharply to about 40 per cent; past 60 years of age, the incidence gradually declined further, and in patients beyond the age of 70, Duroziez's sign of the peripheral type was never obtainable.
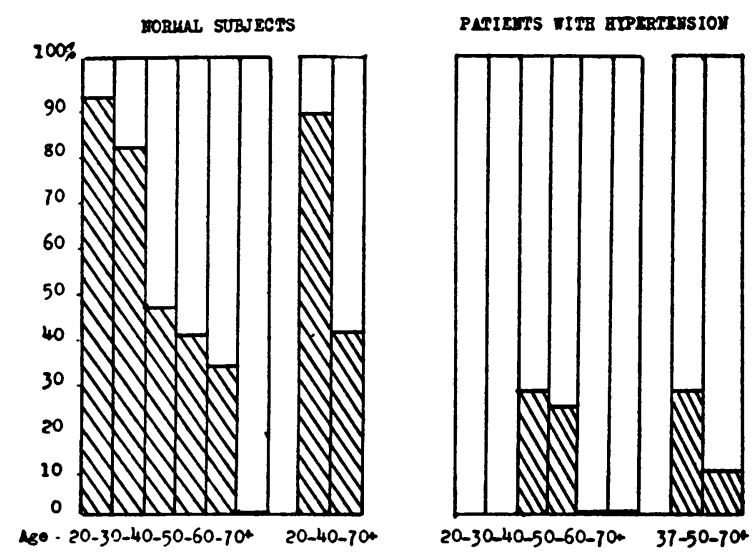

Fig. 2. Duroziez's Sign in Normal Subjects and Patients with Hypertension.

Variation in percentage incidence of Duroziez's sign with age.

${ }^{3}$ For the purpose of this study patients with no evidence of cardiovascular disease are considered normal.
In patients with arterial hypertension the incidence of Duroziez's sign of the peripheral type was generally lower than in subjects with normal arterial pressure; and the incidence declined more sharply toward zero as age advanced (Figure 2). The lowered incidence in arterial hypertension is even more striking when the 42 normal subjects are contrasted with the 41 patients with hypertension of similar ages (Table I).

TABLE I

Incidence of Duroziez's sign according to age

\begin{tabular}{|c|c|c|c|c|c|c|}
\hline \multirow{3}{*}{ Age } & \multicolumn{3}{|c|}{ Normal subjects } & \multicolumn{3}{|c|}{$\begin{array}{l}\text { Patients with arterial } \\
\text { hypertension }\end{array}$} \\
\hline & \multirow{2}{*}{$\begin{array}{l}\text { Number } \\
\text { of } \\
\text { subjects }\end{array}$} & \multicolumn{2}{|c|}{ Positive Duroziez } & \multirow{2}{*}{$-\begin{array}{c}\text { Number } \\
\text { of } \\
\text { subjects }\end{array}$} & \multicolumn{2}{|c|}{ Positive Duroziez } \\
\hline & & Subjects & Per cent & & Subjects & Per cent \\
\hline $\begin{array}{c}\text { years } \\
40 \text { to } 60 \\
60 \text { to } 73 \\
\text { Total and } \\
\text { averages }\end{array}$ & $\begin{array}{r}33 \\
9\end{array}$ & $\begin{array}{r}14 \\
3\end{array}$ & $\begin{array}{l}42 \\
33 \\
\\
40\end{array}$ & $\begin{array}{l}24 \\
17 \\
41\end{array}$ & $\begin{array}{l}6 \\
0\end{array}$ & $\begin{array}{r}25 \\
0 \\
14\end{array}$ \\
\hline
\end{tabular}

Duroziez's sign and pulse pressure. No relation between the magnitude of pulse pressure and the incidence of Duroziez's sign was found in normal persons under the age of fifty. Above that

TABLE II

Incidence of Duroziez's sign according to pulse pressure

\begin{tabular}{|c|c|c|c|c|c|c|c|c|c|}
\hline \multirow{4}{*}{$\begin{array}{c}\text { Pulse } \\
\text { pressure }\end{array}$} & \multicolumn{6}{|c|}{ Normal subjects } & \multirow{2}{*}{\multicolumn{3}{|c|}{$\begin{array}{c}\begin{array}{c}\text { Patients with arte- } \\
\text { rial hypertension }\end{array} \\
\text { All ages }\end{array}$}} \\
\hline & \multirow{2}{*}{\multicolumn{3}{|c|}{$\begin{array}{l}\text { Under } 50 \text { years } \\
\text { Duroziez's sign }\end{array}$}} & \multirow{2}{*}{\multicolumn{3}{|c|}{$\frac{\text { Over } 50 \text { years }}{\text { Duroziez's sign }}$}} & & & \\
\hline & & & & & & & \multicolumn{3}{|c|}{ Duroziez's sign } \\
\hline & + & - & $\begin{array}{c}\text { Percentage } \\
\text { positive }\end{array}$ & + & - & $\begin{array}{c}\text { Per- } \\
\text { centage } \\
\text { positive }\end{array}$ & + & - & $\begin{array}{c}\text { Percentage } \\
\text { positive }\end{array}$ \\
\hline $\begin{array}{l}m m . H g \\
20 \text { to } 40 \\
40 \text { to } 60 \\
60 \text { to } 80 \\
80 \text { to } 100 \\
100+\end{array}$ & $\begin{array}{l}11 \\
\frac{18}{二}\end{array}$ & $\begin{array}{l}6 \\
8 \\
= \\
-\end{array}$ & $\begin{array}{l}64 \\
= \\
=\end{array}$ & $\begin{array}{l}8 \\
0 \\
= \\
-\end{array}$ & $\begin{array}{r}4 \\
10 \\
=\end{array}$ & $\begin{array}{l}66 \\
0 \\
=\end{array}$ & $\begin{array}{l}\overline{4} \\
1 \\
1 \\
0\end{array}$ & $\begin{array}{l}74 \\
10 \\
10 \\
10\end{array}$ & $\begin{array}{r}\overline{50} \\
9 \\
9 \\
0\end{array}$ \\
\hline
\end{tabular}

age, however, and in hypertensive subjects of all age groups, it was found that the greater the pulse pressure, the lower the incidence of Duroziez's sign (Table II).

Duroziez's sign and diastolic blood pressure. 
The incidence of Duroziez's sign in normal subjects with diastolic blood pressures below $80 \mathrm{~mm}$. of mercury was compared with that in normal subjects with pressures above $80 \mathrm{~mm}$. of mercury. Taking into account the influence of age, those with the higher diastolic pressure showed Duroziez's sign more frequently. This relationship was even more pronounced in the patients with arterial hypertension (Table III).

TABLE III

Incidence of Duroziez's sign according to diastolic blood pressure

\begin{tabular}{|c|c|c|c|c|c|c|}
\hline \multirow{3}{*}{$\begin{array}{c}\text { Diastolic } \\
\text { blood } \\
\text { pressure }\end{array}$} & \multicolumn{3}{|c|}{ Normal subjects } & \multicolumn{3}{|c|}{$\begin{array}{c}\text { Patients with arterial } \\
\text { hypertension }\end{array}$} \\
\hline & \multicolumn{3}{|c|}{ Duroziez's sign } & \multicolumn{3}{|c|}{ Duroziez's sign } \\
\hline & + & - & $\begin{array}{l}\text { Percentage } \\
\text { positive }\end{array}$ & + & - & $\begin{array}{l}\text { Percentage } \\
\text { positive }\end{array}$ \\
\hline $\begin{array}{l}m m_{.} H_{g} \\
60-80 \\
80-100 \\
100-120 \\
120+\end{array}$ & $\begin{array}{l}15 \\
20 \\
-\end{array}$ & $\begin{array}{l}15 \\
10 \\
-\end{array}$ & $\begin{array}{l}50 \\
66 \\
- \\
-\end{array}$ & $\begin{array}{l}\overline{0} \\
3 \\
3\end{array}$ & $\begin{array}{r}3 \\
12 \\
13 \\
6\end{array}$ & $\begin{array}{r}0 \\
0 \\
19 \\
33\end{array}$ \\
\hline
\end{tabular}

Duroziez's sign and pulse rate. We found no relation between the radial pulse rate and the incidence of Duroziez's sign. Immersion of the arm had no apparent effect on the pulse rate or on the systolic or diastolic arterial pressure.

\section{B. Capillary pulsation in normal subjects and in patients with arterial hypertension}

Capillary pulsation after immersion. Capillary pulsation was present after immersion in all cases of all groups. This finding differs somewhat from the observation of Lewis (2). He states that there is an obvious, though not wholly constant relation between age and exhibition of capillary pulsation in the heated skin. "Subjects ranging in age from seventeen to thirty-five display capillary pulsation most fully and with much uniformity in its degree. As age advances into and beyond the forties it becomes less evident than in young people and many are found in which it is but slight ... or in which it is altogether absent." We have never found it absent in the heated skin and have found only a slight, if any, relationship between intensity and age.
Incidence of spontaneous capillary pulsation. It is of interest to note the number of subjects showing spontaneous capillary pulsation. Of sixty-three normal subjects, nineteen, or 30 per cent, were positive; of forty-one patients with hypertension twenty-two, or 55 per cent, were positive. Almost all of these patients had normal body temperature and were examined under similar external conditions, the room temperature varying only from $74^{\circ}$ to $76^{\circ} \mathrm{F}$.

Spontaneous capillary pulsation and age. In the normal subjects no relationship between age and the presence of spontaneous capillary pulsation was apparent. In the hypertensive group, however, the older age groups showed a greater incidence of spontaneous capillary pulsation (Figure 3 ). This was attributable not to age, per se,

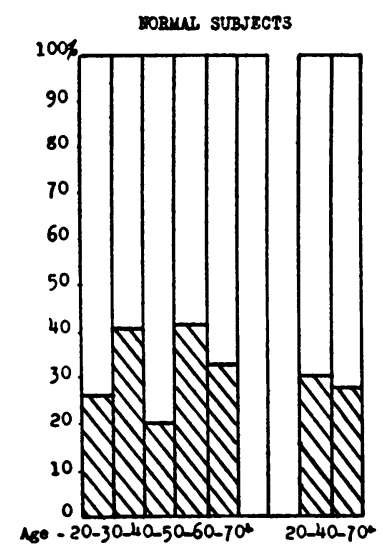

PATIENTS TITE GXPRRTHUS ION

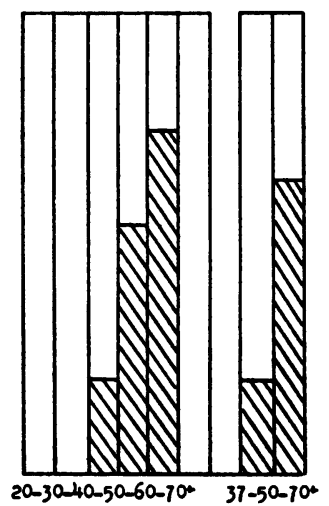

Fig. 3. Capillary Pulsation in Normal Subjects and Patients with Hypertension.

Variation in percentage incidence of spontaneous capillary pulsation with age.

but rather to the fact that the older subjects in the group showed the widest pulse pressures.

Spontaneous capillary pulsation and pulse pressure. Both in the normal and hypertensive subjects the incidence of spontaneous capillary pulsation varied directly with the magnitude of pulse pressure, as seen below.

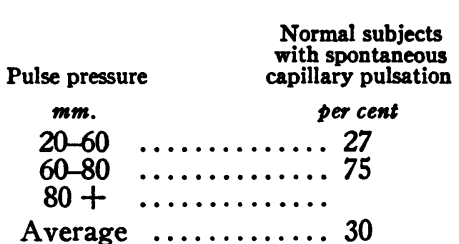

Subjects with arterial hypertension with spontaneous capillary pulsation per cent 30 56 75 55 
The relatively greater incidence of spontaneous capillary pulsation in hypertension is due, we believe, to the wider pulse pressures so frequently seen in this disease.

Spontaneous capillary pulsation and diastolic blood pressure. The height of the diastolic blood pressure per se did not seem to influence the incidence of spontaneous capillary pulsation. In both hypertensive and normal groups spontaneous capillary pulsation was often found in patients with low diastolic pressure. This was probably due to the larger pulse pressures in such subjects.

\section{MISCELLANEOUS GROUP}

In view of the well known fact that the velocity and volume of blood flow frequently are increased in anemia and that this would theoretically predispose to the presence of Duroziez's sign, four cases with primary anemia and two cases with secondary anemia were studied. The three youngest patients showed Duroziez's sign after immersion of the arm and the other three did not.

Two cases of polycythemia vera with hypertension showed no Duroziez's sign, which was in accord with our findings in patients with hypertension. In both, spontaneous capillary pulsation was present.

One case each of thrombo-angiitis obliterans and Raynaud's disease, interestingly enough, showed a positive Duroziez's sign. In both, signs and symptoms of the disease were referable only to the lower extremities.

\section{COMMENT}

Until recently, the sign originally described by Duroziez, namely, a diastolic murmur heard over the larger peripheral arteries, ${ }^{4}$ was always associated with aortic insufficiency and was attributed to a diastolic reflux of blood found in that condition. Of late it has been shown that this sign is not pathognomic of that disease, inasmuch as it has been demonstrated to be present in other conditions, and, in fact, can be artificially produced in normal individuals. Under such artificial con-

\footnotetext{
- Duroziez's original description referred to a diastolic murmur heard over the femoral artery, but clinical usage has sanctioned the wider application of this sign to a diastolic murmur heard over any of the larger peripheral vessels.
}

ditions where aortic reflux is not a factor, Duroziez's sign has been shown to be due to an increase in peripheral blood flow dependent upon the extent of peripheral vasodilatation. To distinguish this type of Duroziez's sign from that present in aortic regurgitation, we have termed it "the peripheral type." When, therefore, Duroziez's sign of the peripheral type is not present spontaneously and cannot be artificially induced, it may be assumed that the peripheral blood flow in the part studied cannot be sufficiently increased. Other factors being constant, either or both of the following situations may be present: (1) the minute vessels cannot dilate sufficiently to permit the increased forward flow of blood necessary to produce Duroziez's sign. (2) The number of minute vessels is decreased, even though those remaining are capable of dilating. The extent of the peripheral vascular bed would thus be decreased. Under such conditions changes in peripheral blood flow might still occur but the blood flow would be insufficient to produce Duroziez's sign. However, Weiss and Frazier (4) have shown that the number of minute vessels of the skin is probably not diminished. The first mechanism would, therefore, seem to be the more important.

The decreasing incidence of Duroziez's sign of the peripheral type with advancing years signifies a gradual reduction in the peripheral vascular bed due either to spasm or sclerosis of the minute vessels. Since local heat used in the elicitation of this sign abalishes spasm, the lowered incidence of Duroziez's sign is presumably due to a relative inability of the minute vessels to dilate or to a decrease in their number. The generally lowered incidence of Duroziez's sign in patients with arterial hypertension is in harmony with the clinical and pathological observations of predominant involvement of the arterioles in that condition.

Our studies were confined to the forearm and, although arteriolar sclerosis in muscle and skin according to certain pathological studies is considered rare, the present clinical studies of Duroziez's sign suggest that the peripheral functional vascular bed is affected to greater degree in such subjects than in similar persons with normal blood pressure.

Three normal subjects failed to show a positive Duroziez's sign, and six patients with arterial 
hypertension in whom the sign was elicited were exceptions to the general findings. Two of the three normal subjects were 39 and 40 years of age and may have had early arteriosclerosis.

Although Duroziez's sign in aortic regurgitation is associated with wide pulse pressure, our observations indicate that in normal subjects above the age of 50 and in hypertensive subjects of all ages the artificially induced Duroziez's sign tends to occur particularly in persons with small pulse pressure; and that it is absent in patients of advanced years who show a wide pulse pressure. This is probably due to the fact that the aging process and hypertension both favor the development of arterial and arteriolar sclerosis. The peripheral resistance becomes increased and the elasticity of the aorta is lessened. Under such circumstances the peripheral vascular tree approximates more closely a rigid system so that the entrance of blood from the heart results in a wide pulse pressure.

The observation that heating the arm of normal subjects to $114^{\circ} \mathrm{F}$. brings out a capillary pulsation confirms the findings of Lewis and other observers (3), who believe that such capillary pulsation is a physiological phenomenon dependent on the state of the local arterioles. Since capillary pulsation, according to our findings, may be elicited in all persons regardless of age, its presence in the heated skin seems of little value as a qualitative test of the condition of the minute vessels. The frequency with which it occurs spontaneously in normal persons and in patients with cardiovascular disease of all types diminishes its diagnostic importance.

From these comparative studies of capillary pulsation and of Duroziez's sign of the peripheral type it is evident that a relatively small increase in peripheral blood flow is sufficient to produce capillary pulsation. Only when blood flow is still further increased does Duroziez's sign appear. Since a greater blood flow is necessary to produce Duroziez's sign, the latter may prove a better clinical gauge of the state of the peripheral minute vessels. Moreover, Duroziez's sign of the peripheral type may be used as a general index of the state of all the minute vessels of the part studied whereas capillary pulsation provides information regarding only those minute vessels displaying the pulsation.

\section{CONCLUSIONS AND SUMMARY}

1. In 65 subjects with no evidence of cardiovascular disease the incidence of Duroziez's sign decreased with advancing years. This indicates a progressive inability of the peripheral minute vessels to dilate sufficiently as age advances to provide the increased diastolic forward flow of blood necessary to produce the sign.

2. The incidence of the sign was studied in 41 subjects with clinical hypertension. Compared to normal subjects of similar age groups, the incidence was in general lower, particularly with advancing age. This is in accord with the theory that arteriolosclerosis is associated with arterial hypertension.

3. Subjects with wide pulse pressures generally showed a low incidence of Duroziez's sign of the peripheral type. This probably is due to the general coexistence of arterial and arteriolosclerosis.

4. Capillary pulsation was studied both immediately before and after immersion of the arm in water at $114^{\circ} \mathrm{F}$. Of 61 subjects with no evidence of vascular disease 31 per cent exhibited it spontaneously. Of 41 cases with clinical hypertension 54 per cent showed a spontaneous capillary pulsation. There was no apparent relation to age.

5. Capillary pulsation was particularly evident in subjects with wide pulse pressures. The greater incidence of spontaneous capillary pulsation in the hypertensive group was probably due to the greater pulse pressure generally seen in this disease.

6. The height of diastolic blood pressure seemed in no way to influence the incidence of spontaneous capillary pulsation except insofar as the height of diastolic pressure affected the magnitude of pulse pressure.

7. Capillary pulsation after immersion at $114^{\circ}$ F. was present in every case. The ages of the subjects ranged from twenty to seventy-three years. Capillary pulsation, since it was always present in the heated skin, regardless of age, cannot be used as a qualitative test of the condition of the peripheral arterioles.

8. Duroziez's sign produced in the manner described may be used to throw light on the general condition of the peripheral arterioles. According to our findings, any person under the age of thirty-five who fails to show a Duroziez's sign 
after immersion of the arm in water at $114^{\circ} \mathrm{F}$. digresses from the physiologic norm in that his functional peripheral vascular bed is relatively fixed.

\section{BIBLIOGRAPHY}

1. Blumgart, H. L., and Ernstene, A. C., Two mechanisms in the production of Duroziez's sign. J. A. M. A., 1933, 100, 173.

2. Lewis, Thomas, Studies of capillary pulsation with special reference to vasodilatation in aortic regurgitation and including observations on the effects of heating the human skin. Heart, 1924, 11, 151.

3. Bernard, Claude, Sur les variations de couleur dans le sang veineux des organes glandulaires suivant leur état de fonction ou de repos. J. d. 1. Physiol. d l'homme e. d. animaux, 1858, 1, 233.

4. Weiss, S. and Frazier, W. R., The density of the surface capillary bed of the forearm in health, in arterial hypertension, and in arteriosclerosis. Am. Heart J., 1930, 5, 511. 